\title{
SYSTEMATIC DESIGN OF MEMS RESONATORS FOR OPTIMAL NONLINEAR DYNAMIC RESPONSE
}

\author{
Lily L. Li ${ }^{l^{*}}$, Pavel M. Polunin ${ }^{2}$, Suguang Dou ${ }^{3}$,Oriel Shoshani ${ }^{4}$, B. Scott Strachan ${ }^{2,5}$, \\ Jakob S. Jensen ${ }^{3}$, Steven W. Shaw ${ }^{2,4}$ and Kimberly L. Turner ${ }^{1}$ \\ ${ }^{1}$ University of California, Santa Barbara, CA 93106 USA \\ ${ }^{2}$ Michigan State University, East Lansing, MI 48824 USA \\ ${ }^{3}$ Technical University of Denmark, Kgs. Lyngby, 2800 Denmark \\ ${ }^{4}$ Florida Institute of Technology, Melbourne, FL 32901 USA \\ ${ }^{5}$ SiTime Corporation, Sunnyvale, CA 94085 USA
}

\begin{abstract}
We report on the first experimental demonstration of an effective and systematic control over the mechanical contribution to the Duffing nonlinearity in microelectromechanical (MEMS) resonators using nonlinear shape optimization methods. A set of microbeams with selected variable geometry profiles optimized for in-plane vibrations was designed and characterized. Experimental results have demonstrated that these shape changes result in more than a three-fold increase and a two-fold reduction in the Duffing nonlinearity due to resonator mid-line stretching. The minimization of the cubic term in the resonator stiffness resulted in a substantial increase of the resonator linear range.
\end{abstract}

\section{INTRODUCTION}

Resonant micro-electromechanical-systems (MEMS) have grown in popularity over the past several decades due to their numerous applications including stable frequency generation and timing [1-2], robust mass and angular rate sensing [3-4], precise signal filtering [5-6], and energy harvesting [7]. While the majority of MEMS resonators are designed to operate in their linear dynamic range, research has shown that utilizing nonlinearity in resonant MEMS sensors can significantly improve the performance of these systems in some of the applications mentioned above [1,8]. Nonlinear stiffness effects in MEMS resonators commonly arise from finite deformations that lead to nonlinear strain-displacement relationships [9], and/or the nonlinear nature of electrostatic forces in capacitive MEMS [4-6, 10-11].

The ability to control the nonlinearity in resonant MEMS allows one to design devices based on their applications with greater control over their dynamical responses. It has been shown that one can independently tune the linear and/or cubic stiffness coefficients due to the electrostatic potential of the capacitive drive in order to relax the constraints in mode mismatch in MEMS gyroscopes due to fabrications errors $[4,12]$, to achieve optimal drive conditions for micro resonators [13], and to enhance their dynamic range [14]. On the other hand, Dou et al. manipulated geometric contributions in the nonlinear resonator stiffness parameters by performing the shape optimization of the thickness profile in different MEMS resonators to achieve targeted nonlinear coefficients using a gradient-based optimization method [15-16]. The numerical results in $[15,17]$ showed that removing/adding the material (by changing the cross-sectional area) from the points where the slope of the resonator mode shape is maximal results in continuous decrease/increase of the geometric contribution to the resonator cubic nonlinearity. This effect becomes more pronounced as the difference in the beam thickness increases. We followed the shape optimization approach described in [15] in order to manipulate the nonlinearity in a set of clampedclamped microbeams, and selected a few designs with geometries corresponding to different stages of the resonator shape optimization process. The beams were fabricated in single-crystal silicon using standard SOI (silicon-on-insulator) processing techniques with deep reactive ion etching and released with HF vapor. Figure 1 shows SEM images and COMSOL models of three of representative designs of the clamped-clamped beams under study: a beam with uniform thickness (Beam_initial) and beams designed to minimize/maximize ${ }^{-}$the cubic nonlinearity (Beam_min/Beam_max). The resonant frequencies of the beams range from 80 to $\overline{200} \mathrm{kHz}$.

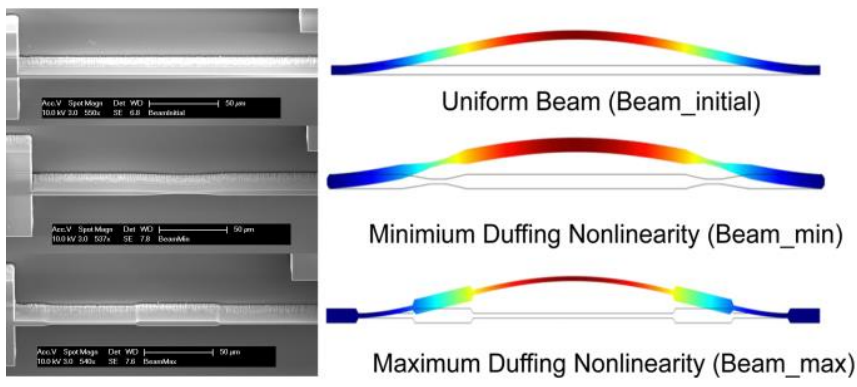

Figure 1: SEM images and COMSOL models of three representative clamped-clamped microbeams under study. "Beam_initial" is the beam with uniform thickness, while "Beam_min"/"Beam_max" are designed to minimize/maximize the resonator Duffing nonlinearity. Beam dimensions are 500 um (length) by 20 um (out-of-plane thickness) by 2 6 $\mu \mathrm{m}$ (inplane thickness).

\section{THEORY}

The dynamic response of a clamped-clamped microbeam performing in-plane flexural vibrations can be modeled by the standard Duffing equation with positive cubic stiffness coefficient [18-19] as follows:

$$
\ddot{x}+Q^{-1} \omega_{0} \dot{x}+\omega_{o}^{2} x\left(1+\gamma x^{2}\right)=f \cos (\omega t)
$$

where $\omega_{0}$ is the natural frequency of the beam, $Q$ is the quality factor, $\gamma$ is the coefficient of the cubic nonlinear stiffness term (Duffing nonlinearity), and $f$ and $\omega$ are the drive amplitude and frequency. The critical vibration amplitude $\left(a_{n l}\right)$ at which

Solid-State Sensors, Actuators and Microsystems Workshop Hilton Head Island, South Carolina, June 5-9, 2016 
nonlinear effects result in a qualitative change of the resonator response, specifically, at which the frequency response transitions from being single-valued to multi-valued, is given by:

$$
a_{n l}=\left(\frac{64}{27}\right)^{1 / 4} \frac{1}{\sqrt{Q \gamma}}
$$

which shows that one can maximize the resonator critical vibration amplitude by reducing the Duffing nonlinearity $\gamma$ [18]. Once the system response enters its nonlinear region, the relationship between the nonlinear resonance amplitude $\left(a_{p}\right)$ and frequency $\left(\omega_{p}\right)$ can be written as:

$$
\omega_{p}\left(a_{p}\right)=\omega_{0}\left(1+\frac{3}{8} \gamma a_{p}^{2}\right)
$$

Equation (3) provides the so-called Duffing backbone curve [19] that characterizes the system nonlinearity in the form of amplitude-frequency dependence. In order to compare the results of the shape optimization method on the Duffing nonlinearity of the system, we use the effective Duffing nonlinearity $\gamma_{e f f}=\frac{3}{8} \gamma$ as the objective function to be minimized/maximized.

\section{EXPERIMENTS}

In the experiment, the beams were externally actuated by a shear piezoelectric stack actuator to achieve in-plane excitation. The in-plane motion of each beam was detected using a Laser Doppler Vibrometer by tilting the microbeams at $45^{\circ}$ angle with the laser focused on the sidewalls. The actual in-plane velocity of the microbeam can be calculated from simple geometry. This
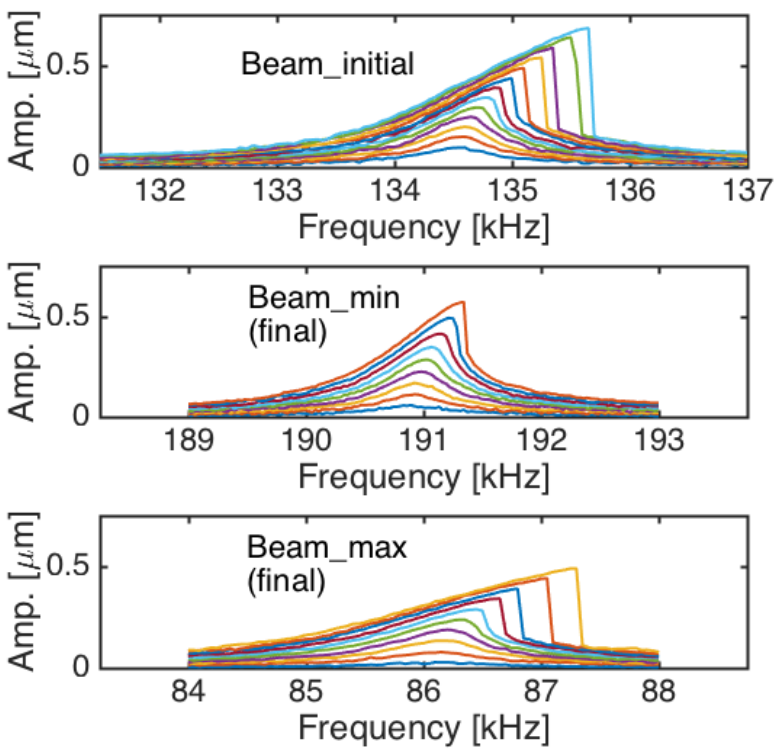

Figure 3: Amplitude-frequency responses of microbeams exhibit hardening behavior. The drive voltages for the three beams from top to bottom are: 0.2:0.1:1.4 V, 0.7:0.5:4.7 $\mathrm{V}$ and 0.2:0.4:4.0 V respectively. arrangement allowed characterization of the mechanical nonlinearity isolated from electrostatic effects. Amplitudefrequency responses for each beam were obtained at multiple drive amplitudes in atmospheric pressure from a spectrum analyzer. The schematic in Figure 2 depicts the experimental setup.

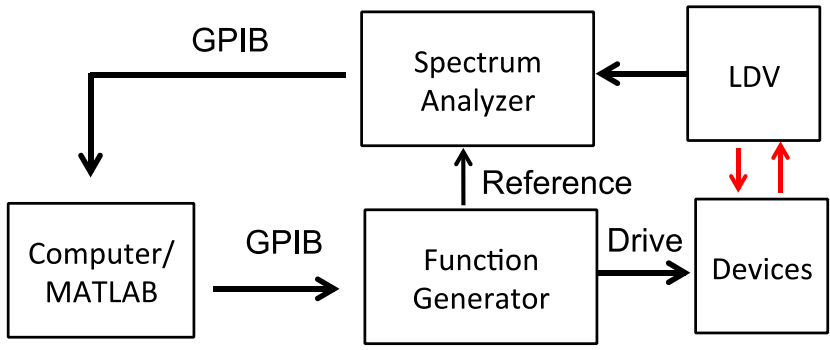

Figure 2: Experimental setup schematic. Microbeams are actuated externally by a shear piezoelectric stack. The in-plane responses are measured with a Laser Doppler Vibrometer (LDV, Polytec MSA-400) by tilting the beams at $45^{\circ}$ with the laser focused on the sidewalls. The amplitude-frequency response curves are obtained from the spectrum analyzer (HP 88410A).

\section{RESULTS}

The amplitude-frequency responses for the three representative beams shown in Figure 1 are plotted in Figure 3. As the driving amplitude increases, the amplitude-frequency response of each microbeam exhibits hardening behavior, as expected from a positive Duffing nonlinearity due to the midline stretching effects. Once the system response enters its

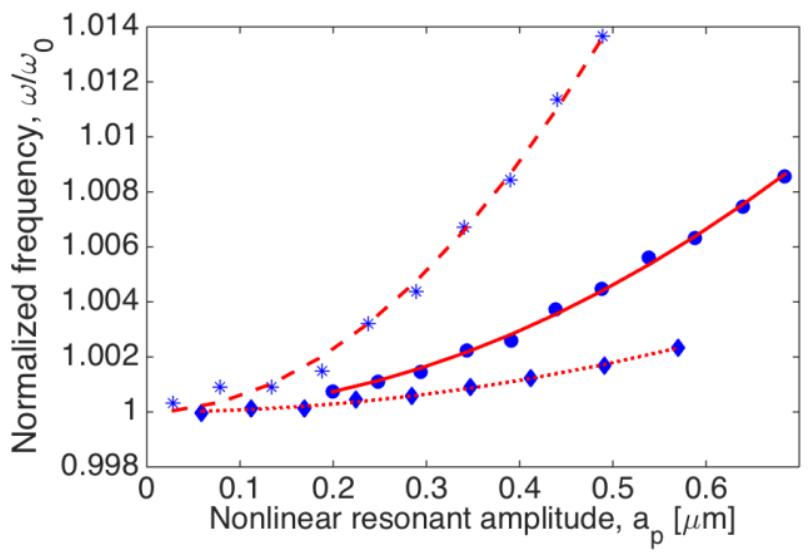

Figure 4: Amplitude-frequency characteristics of three representative microbeams. Solid, dotted and dashed curves represent least-square fitting of experimental results for "beam_initial", "beam_min" (final), and "beam_max" (final) respectively. 
Table 1: Characterization results for microbeams under study. Data shows clear trends of the Duffing nonlinearity towards its minimum/maximum values depending on the beam geometry profile. The discrepancy between numerical and experimental results for the beams designed to maximize $\gamma_{\text {eff }}$ is expected due to abrupt changes in the beam thickness profile, which causes errors in the numerical algorithm utilizing simple beam elements. In contrast, the relative error between numerical and experimental results for the beams designed to minimize $\gamma_{\text {eff }}$ is $8 \%$ or less due to comparatively smooth beam profiles.

\begin{tabular}{|c|c|c|c|c|c|c|c|}
\hline \multirow{2}{*}{ Beam type } & $\begin{array}{l}\text { COMSOL } \\
\text { Results }\end{array}$ & \multicolumn{3}{|c|}{$\begin{array}{c}\text { Numerical Results } \\
\text { (following techniques in [15]) }\end{array}$} & \multicolumn{3}{|c|}{$\begin{array}{c}\text { Experimental Results } \\
\text { (from least-square curve fits) }\end{array}$} \\
\hline & $\frac{\omega_{o}}{2 \pi}, \mathrm{kHz}$ & $\frac{\omega_{o}}{2 \pi}, \mathrm{kHz}$ & $\gamma_{e f f}, \mu m^{-2}$ & $\frac{\gamma_{e f f}}{\gamma_{e f f, 0}}$ & $\frac{\omega_{o}}{2 \pi}, \mathrm{kHz}$ & $\gamma_{e f f}, \mu m^{-2}$ & $\frac{\gamma_{e f f}}{\gamma_{e f f, 0}}$ \\
\hline Beam_initial & 135.0 & 135.1 & 0.0165 & 1 & 134.5 & 0.0188 & 1 \\
\hline Beam min $\left(5^{\text {th }}\right.$ iteration $)$ & 145.7 & 148.7 & 0.0117 & 0.712 & 145.0 & 0.0129 & 0.699 \\
\hline Beam_min (Final) & 191.9 & 189.4 & 0.0059 & 0.357 & 190.9 & 0.0072 & 0.388 \\
\hline Beam_max $\left(20^{\text {th }}\right.$ iteration $)$ & 86.8 & 85.8 & 0.0844 & 5.111 & 91.8 & 0.0472 & 2.558 \\
\hline Beam_max (Final) & 79.9 & 70.5 & 0.1248 & 7.559 & 86.1 & 0.0569 & 3.309 \\
\hline
\end{tabular}

bistable regime, the maximum nonlinear response amplitude is only a function of its corresponding frequency, as described by equation (3). Using this result, we extracted the peak amplitude from Figure 3 and plotted the frequency as a function of the peak amplitude $\left(a_{p}\right)$, followed by curve fitting to obtain the natural frequency and cubic nonlinearity. Quadratic curve fitting is performed to the data using $\omega\left(a_{p}\right)=\boldsymbol{a}\left(1+\boldsymbol{b} a_{p}^{2}\right)$, where the fitting coefficients " $\boldsymbol{a}$ " and " $\boldsymbol{b}$ " correspond to the beam natural frequency $\omega_{0}$ and the effective Duffing nonlinearity $\gamma_{\text {eff }}$ respectively. After the coefficients are determined, we normalized the frequency of each beam design to its natural frequency for direct comparison of the three representative beams (Figure 4). We further compared the experimental results to the numerical simulations following the process in [15]. Table 1 summarizes the comparisons between the beam designs, including the intermediate ones.

\section{DISCUSSION}

We compared the values of $\gamma_{\text {eff }}$ for beams at different stages of the optimization iteration process with the corresponding Duffing nonlinearity of the nominal (uniform) beam, $\gamma_{e f f, 0}$; the results are summarized in Table 1 . These characterization results show that the resonator shape optimization results in the natural frequency varying from its nominal, uniform beam, value. They also demonstrate that the designs to minimize nonlinearity reduce the Duffing nonlinearity by a factor of 2.6 when compared to the initial uniform beam, which corresponds to a 1.6 times increase in the critical vibration amplitude at which the resonator becomes nonlinear [18]. Meanwhile, the beam design with maximum nonlinearity achieves a 3.3 times increase in the Duffing nonlinearity compared to the uniform beam. Further, we performed numerical simulations on the same beam geometries using the optimization techniques described in [15]. When compared the experimental results to the numerical predictions, the minimization of $\gamma_{e f f}$ agrees with the simulations within $8 \%$. A larger discrepancy occurs in the maximization of $\gamma_{e f f}$, however, the trend qualitatively agrees with the numerical results. The difference between numerical and experimental results for the beams designed to maximize $\gamma_{\text {eff }}$ is due to the abrupt changes in the beam thickness profile, which causes errors in the numerical algorithm, which utilizes simple beam elements. In contrast, beams designed to minimize $\gamma_{\text {eff }}$ results in relatively small errors due to the relatively smooth beam profiles.

\section{CONCLUSION}

The experimental results shown in this work are in good agreement with numerical predictions [15] for the important case of minimizing the nonlinearity, and they demonstrate that one can successfully utilize shape optimization methods for adjusting the resonator nonlinearity in a well-controlled manner. The ability of reducing the Duffing nonlinearity is especially useful in increasing the linear dynamical range of resonators, which plays a pivotal role in reducing phase noise in MEMS oscillators and for increasing the signal to noise ratio in resonant sensors [20]. Finally, our results provide strong confidence that shape optimization methods can be applied in the design of nonlinear MEMS resonators with more complicated geometries, with multi-physics effects, and for different nonlinear parameters, including hard-to-control modal coupling coefficients [15].

\section{ACKNOWLEGEMENT}

This work was supported by NSF grant 442550-22031FGKT10-2, CMU/DAPPA grant 8-442550-59085-FGKT11-2 and ERC Starting grant 279529 (INNODYN). The content of the information does not necessary reflect the position or the policy of the Government and no official endorsement should be inferred. Travel support has been generously provided by the Transducer Research Foundation. In addition, the authors would like to thank Brian Gibson for his support in fabrications.

\section{REFERENCES}

[1] Antonio, D., Zanette, D. H., \& López, D. (2012). Frequency stabilization in nonlinear micromechanical oscillators. Nature communications, 3, 806 .

[2] Hopcroft, M. A., Lee, H. K., Kim, B., Melamud, R., 
Chandorkar, S., Agarwal, M., ... \& Kenny, T. W. (2007, June). A high-stability MEMS frequency reference. In Solid-State Sensors, Actuators and Microsystems Conference, 2007. TRANSDUCERS 2007. International (pp. 1307-1309). IEEE.

[3] Li, L. L., Holthoff, E. L., Shaw, L. A., Burgner, C. B., \& Turner, K. L. (2014). Noise squeezing controlled parametric bifurcation tracking of MIP-coated microbeam MEMS sensor for TNT explosive gas sensing. Journal of Microelectromechanical Systems, 23(5), 1228-1236.

[4] Rhoads, J. F., Shaw, S. W., \& Turner, K. L. (2010). Nonlinear dynamics and its applications in micro-and nanoresonators. Journal of Dynamic Systems, Measurement, and Control, 132(3), 034001.Lin, L., Howe, R. T., \& Pisano, A. P. (1998). Microelectromechanical filters for signal processing. Microelectromechanical Systems, Journal of, 7(3), 286294.

[5] Oropeza-Ramos, L. A., Burgner, C. B., \& Turner, K. L. (2009). Robust micro-rate sensor actuated by parametric resonance. Sensors and Actuators A: Physical, 152(1), 80-87.

[6] Shaw, S. W., Turner, K. L., Rhoads, J. F., \& Baskaran, R. (2005). Parametrically excited MEMS-based filters. In IUTAM Symposium on Chaotic Dynamics and Control of Systems and Processes in Mechanics (pp. 137-146). Springer Netherlands.

[7] Challa, V. R., Prasad, M. G., Shi, Y., \& Fisher, F. T. (2008). A vibration energy harvesting device with bidirectional resonance frequency tunability. Smart Materials and Structures, 17(1), 015035.

[8] Nitzan, S. H., Zega, V., Li, M., Ahn, C. H., Corigliano, A., Kenny, T. W., \& Horsley, D. A. (2015). Self-induced parametric amplification arising from nonlinear elastic coupling in a micromechanical resonating disk gyroscope. Scientific reports, 5 .

[9] Cho, H., Jeong, B., Yu, M. F., Vakakis, A. F., McFarland, D. M., \& Bergman, L. A. (2012). Nonlinear hardening and softening resonances in micromechanical cantilever-nanotube systems originated from nanoscale geometric nonlinearities. International Journal of Solids and Structures, 49 (15), 2059-2065.

[10] Tatar, E., Mukherjee, T., \& Fedder, G. K. (2015, June). Nonlinearity tuning and its effects on the performance of a MEMS gyroscope. In Solid-State Sensors, Actuators and Microsystems (TRANSDUCERS), 2015 Transducers-2015 18th International Conference on (pp. 1133-1136). IEEE.

[11] Guo, C., \& Fedder, G. K. (2013). A quadratic-shapedfinger comb parametric resonator. Journal of Micromechanics and Microengineering, 23(9), 095007.

[12] DeMartini, B. E., Rhoads, J. F., Turner, K. L., Shaw, S. W., \& Moehlis, J. (2007). Linear and nonlinear tuning of parametrically excited MEMS oscillators. Microelectromechanical Systems, Journal of, 16(2), 310318.

[13] Agarwal, M., Chandorkar, S. A., Candler, R. N., Kim, B., Hopcroft, M. A., Melamud, R.,Jha, C.M., Kenny, T.W. \& Murmann, B. (2006). Optimal drive condition for nonlinearity reduction in electrostatic microresonators. Applied physics letters, 89(21), 214105.

[14] Saghafi, M., Dankowicz, H., \& Lacarbonara, W. (2015, July). Nonlinear tuning of microresonators for dynamic range enhancement. In Proc. $R$. Soc. $A$ (Vol. 471, No. 2179 , p. 20140969). The Royal Society.

[15] Dou, S., Strachan, B.S., Shaw, S.W. and Jensen, J.S., 2015. Structural optimization for nonlinear dynamic response. Phil. Trans. R. Soc. A, 373 (2051), p. 20140408.

[16] Dou, S. (2015). Gradient-based optimization in nonlinear structural dynamics (Doctoral dissertation, Technical University of Denmark, Department of Mechanical Engineering).

[17] Dou, S. and Jensen, J.S., 2015. Optimization of nonlinear structural resonance using the incremental harmonic balance method. Journal of Sound and Vibration, 334, pp.239-254.

[18] Lifshitz, R. and Cross, M.C., 2008. Nonlinear dynamics of nanomechanical and micromechanical resonators. Review of nonlinear dynamics and complexity, 1, pp.1-52.

[19] Nayfeh, A.H. and Mook, D.T., 2008. Nonlinear oscillations. John Wiley \& Sons.

[20] Yurke, B., Greywall, D. S., Pargellis, A. N., \& Busch, P. A. (1995). Theory of amplifier-noise evasion in an oscillator employing a nonlinear resonator. Physical Review A, 51(5), 4211.

\section{CONTACT}

*L.L. Li, lily@engr.ucsb.edu 